\title{
JD8 - Hot Interstellar Matter in Elliptical Galaxies
}

\author{
Dong-Woo Kim ${ }^{1}$ and Silvia Pellegrini ${ }^{2}$ \\ ${ }^{1}$ Smithsonian Astrophysical Observatory, 60 Garden Street, Cambridge, MA 02138, USA \\ email: kim@cfa.harvard.edu \\ ${ }^{2}$ Department of Astronomy, University of Bologna, via Ranzani 1, 40127 Bologna, Italy \\ email: silvia.pellegrini@unibo.it
}

\section{Preface}

The physical properties of the hot interstellar matter in elliptical galaxies are directly related with the formation and evolution of elliptical galaxies via star formation episodes, environmental effects such as stripping, infall, and mergers, and growth of super-massive black holes. The recent successful Chandra and XMM-Newton X-ray space missions have provided a large amount of high spatial/spectral resolution observational data on the hot ISM in elliptical galaxies. At the same time, theoretical studies with numerical simulations and analytical modeling of the dynamical and chemical evolution of elliptical galaxies have made a significant progress and start to predict various observable quantities.

As an example of rich sub-structures in the hot ISM, Chandra X-ray (blue \& white) and optical (grey \& white) images of a few elliptical galaxies are shown below (taken from http://chandra.harvard.edu/photo/2006/galaxies; Credit X-ray: NASA/CXC/U. Ohio/T. Statler \& S. Diehl; Optical: DSS). In contrast to the stellar optical light which is smoothly distributed as known for elliptical galaxies, the shapes of the massive clouds of $\mathrm{X}$-ray emitting gas reveal complex structures, indicating that a powerful source of energy must be pushing the hot gas around and stirring it up.

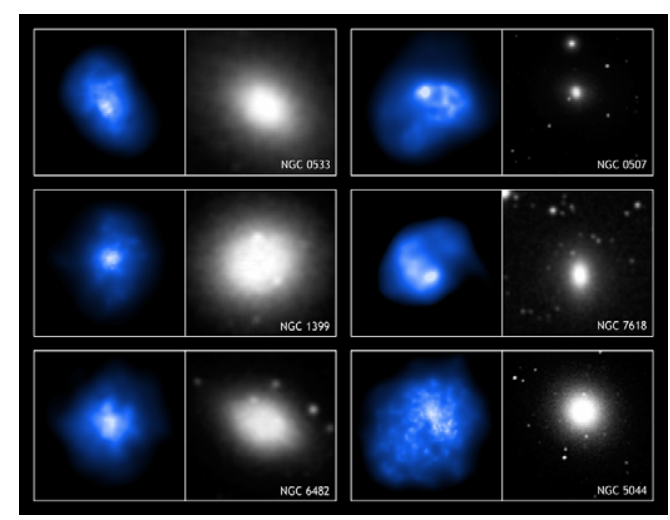

This Joint Discussion between "Galaxies" and "ISM" divisions/commissions was organized during the IAU General Assembly to bring together both observers and theorists in the field. Throughout this JD, we have discussed recent results on the hot interstellar 
matter in elliptical galaxies to identify important, but unsolved problems for further investigations with special emphasis on the spectral and spatial properties of the hot ISM and the comparison with the state-of-the-art theoretical models. During the one and a half day session, 23 speakers, including 4 reviewers, presented their recent achievements.

We thank all the speakers for their excellent presentations, 3 session chairs for timely managing talks and $\mathrm{Q} / \mathrm{A}$, the SOC members for their help in various organizational issues and the local organizers for their supports throughout the preparation.

Dong-Woo Kim and Silvia Pellegrini, SOC co-chairs, Cambridge and Bologna, November 30, 2009

Scientific Organizing Committee

Françoise Combes (France),

Sofia A. Cora (Argentina), Giuseppina Fabbiano (USA), Alexis Finoguenov (Germany),

Brad K. Gibson (UK),

Nimisha G. Kantharia (India),

Dong-Woo Kim (USA, co-Chair),

Chiaki Kobayashi (Japan),

Cludia L. Mendes de Oliveira (Brasil),

Silvia Pellegrini (Italy, co-Chair),

Elaine M. Sadler (Australia),

Craig L. Sarazin (USA),

Thomas S. Statler (USA),

Ginevra Trinchieri (Italy) 